\title{
A low-temperature Kerr effect microscope for the simultaneous magneto-optic and magneto-transport study of magnetic topological insulators
}

\author{
Jieyi Liu ${ }^{1}$ ฯ, Angadjit Singh ${ }^{1}$, ฯ, Justin Llandro ${ }^{2}$, Liam B Duffy ${ }^{3}$, \\ Michael R Stanton ${ }^{1}$, Stuart N Holmes ${ }^{1}$, Matthew J Applegate ${ }^{1}$, \\ Richard T Phillips ${ }^{1}$, Thorsten Hesjedal ${ }^{3}$, and Crispin $\mathbf{H}$ W \\ Barnes $^{1}$ \\ ${ }^{1}$ Cavendish Laboratory, University of Cambridge, J J Thomson Avenue, Cambridge, \\ CB3 0HE, UK \\ ${ }^{2}$ Laboratory for Nanoelectronics and Spintronics, Research Institute of Electrical \\ Communication, Tohoku University, 2-1-1 Katahira, Aoba-ku, Sendai, 980-8577, \\ Japan \\ ${ }^{3}$ Clarendon Laboratory, Department of Physics, University of Oxford, Oxford, \\ OX1 3PU, UK \\ E-mail: j1807@cam.ac.uk
}

\begin{abstract}
Magneto-optical Kerr effect (MOKE) microscopy is a surface-sensitive probe of magnetisation with micron-sized lateral resolution. Here, we present a low-temperature, focused polar MOKE microscope for the simultaneous magnetooptical and magneto-transport measurements, which has a temperature range of 1.6$300 \mathrm{~K}$ and is equipped with a magnet capable of delivering a field of up to $9 \mathrm{~T}$. In this microscope, all optical components are integrated in a free-standing probe, allowing for the straightforward incorporation into many non-optical cryostat systems. Two-dimensional magnetisation scans on patterned ferromagnetic $[\mathrm{CoFeB} / \mathrm{Pt}]_{n}$ films demonstrate a magnetisation sensitivity of $10 \mu \mathrm{rad}$ (Kerr angle) and a spatial resolution of $2.2 \mu \mathrm{m}$. The combination of optical and electrical measurements provides complementary temperature-dependent information, as demonstrated by the study of magnetic topological insulator thin films with out-of-plane magnetic anisotropy. Using this complementary approach, we study the effects of a secondary phase in $\mathrm{Cr}$ and $\mathrm{V}$ co-doped $\mathrm{Sb}_{2} \mathrm{Te}_{3}$ thin films, which show a combination of weak antilocalization and anisotropic magnetoresistance effects above $70 \mathrm{~K}$. Our results highlight the virtue of MOKE and electrical transport to optimise exotic topological magnetic materials, paving the way for energy-efficient spintronic devices.
\end{abstract}

PACS numbers:

Submitted to: Meas. Sci. Technol.

I Both authors contributed equally to this work. 


\section{Introduction}

The magneto-optical Kerr effect (MOKE) describes the changes of reflected light, usually laser light, due to a spin polarised surface. Microscopically, this effect originates from the interaction between the electric field of the light and the electron spins of the material due to spin-orbit coupling. Macroscopically it can be explained by the presence of antisymmetric, off-diagonal elements in the dielectric tensor [1,2]. MOKE is a non-invasive magnetisation measurement technique that combines the advantages of $\mu \mathrm{m}$-sized lateral resolution and surface sensitivity due to the controlled spatial laser profile and its limited penetration depth into a sample $(\sim 20 \mathrm{~nm})$, making it a powerful tool to investigate magnetic thin films and micro-structures. In contrast, many other magnetometers, such as superconducting quantum interference device (SQUID) and vibrating sample magnetometer (VSM) based techniques, are sensitive to bulk materials only. The minimum spot size of a MOKE microscope is diffraction-limited to about $500 \mathrm{~nm}$ using visible light $[3,4]$, however, the magnetisation of nanostructures can be detected using MOKE well below the optical resolution limit [5]. Single-pass hysteresis loops of 30-nm-wide Co nanowires have been successfully detected by MOKE magnetometry [6], demonstrating a measurement sensitivity comparable to that of the latest SQUID magnetometers $\left(10^{-12}\right.$ to $\left.10^{-13} \mathrm{Am}^{2}\right)$. Utilising the spatial resolving power, MOKE microscopy has also been used to resolve the spin Hall effect in metals and semiconductors [7-9], to map spin injection and drift in ferromagnet/semiconductor heterostructures $[10,11]$, to verify ferromagnetism in two-dimensional van der Waals crystals $[12,13]$, and to trace non-monotonic spin relaxation in perpendicular synthetic ferrimagnets [14].

Many materials for which MOKE studies are of interest need to be measured at low temperatures, e.g. due to low Curie temperatures or temperature-dependent spin lifetimes required to resolve spin distributions spatially [7, 9-11]. Although a room temperature MOKE microscope is a common apparatus in magnetism research facilities $[4,5,15]$, due to the challenges associated with incorporating optical instruments into cryogenic systems, it is less common to find low-temperature microscopes that combine the virtues of high sensitivity, high spatial resolution, high magnetic field, and electrical measurement capability. Most low-temperature MOKE magnetometers are designed with optical cryostats in mind, allowing the specimen to be placed on a cold-finger close to a transparent window, while all optical components of the Kerr microscope can stay at ambient conditions $[7,9-11,16,17]$. This is not a feasible approach for users of conventional cryostats specialised in electrical transport measurements, which do not have a direct optical access to the sample from ambient environment. Meanwhile, there has been a growing interest in combining low-temperature electrical transport and magneto-optic studies on various two-dimensional and three-dimensional materials [18-21]. In order to execute combined measurements of this kind, many transport research groups may choose to collaborate with optical groups, or to use fully-commercialised magneto-optic solutions with a large financial cost. It is therefore 
beneficial to develop cost-effective optical instruments that are retro-fitted to many existing transport cryostats, to measure the Kerr effect simultaneously with other important electrical characteristics.

MOKE has been applied to study magnetism on various doped topological insulator (TI) materials [22-25]. TIs refer to materials with electrically insulating bulk, but conducting, topologically protected surface states. The bulk has a finite energy gap, while the surface states, which are gapless due to strong spin-orbit coupling, are protected by time-reversal symmetry (TRS) [26, 27]. Magnetic doping breaks TRS in a TI material $[28,29]$, leading to the realisation of quantum anomalous Hall effect (QAHE) at low temperatures, an energy dissipationless transport phenomenon in the absence of an external magnetic field [30]. QAHE was first observed in Cr-doped and $\mathrm{V}$-doped (Bi,Sb) ${ }_{2} \mathrm{Te}_{3}$ epitaxial thin films below $100 \mathrm{mK}[31]$, then recently in Cr-V-codoped (Bi,Sb) ${ }_{2} \mathrm{Te}_{3}$ at a higher temperature [32], showing a conductance value close to the quantum regime at $1.5 \mathrm{~K}$. This finding brings scientific interest to pursue the co-doping of other compounds in the $(\mathrm{Bi}, \mathrm{Sb})_{2}(\mathrm{Te}, \mathrm{Se})_{3}$ family to realise the QAHE at elevated temperatures [33]. Moreover, the recent discovery of the topological Hall effect (THE) in TI heterostructures highlights the virtue of a combined technique of MOKE and electrical transport $[34,35]$. In this case the magnitude of the THE can be derived from a direct subtraction of the anomalous Hall effect (AHE) results by the Kerr responses $[18,21]$. It is therefore important to examine systematically the consistency between data obtained from anomalous Hall effect (AHE) and MOKE in various magnetic TI (MTI) materials [36].

Here, we present the design and construction of a low-temperature, focused polar MOKE microscope with simultaneous electrical measurement capability, which has a temperature range of $1.6-300 \mathrm{~K}$ and is equipped with a $9 \mathrm{~T}$ magnet. This microscope is a stand-alone optical probe compatible with many conventional non-optical cryostats. We find the lateral resolution of the microscope to be $2.2 \mu \mathrm{m}$, and the detection sensitivity reaches $10 \mu \mathrm{rad}$ (Kerr angle), by performing two-dimensional scans on micro-patterned $[\mathrm{CoFeB} / \mathrm{Pt}]_{n}$ films with out-of-plane magnetic anisotropy. Our study shows the capability to build a high-performance, liquid He Kerr microscope using a non-optical cryogenic facility. The design of this MOKE microscope provides a cost-effective solution for transport cryostat users, without the necessity of purchasing fully-developed commercial magneto-optic systems. Utilising this probe we further conducted a comparison study of Cr-V-co-doped $\mathrm{Sb}_{2} \mathrm{Te}_{3}$ and Cr-doped $\mathrm{Sb}_{2} \mathrm{Te}_{3} \mathrm{MTI}$ thin films by simultaneous MOKE and electrical transport measurements. On both materials, we confirmed out-of-plane magnetisation showing a good consistency between the electrically and optically probed magnetisation characteristics. We also discovered the presence of a secondary, ferromagnetic $\mathrm{Cr}_{2} \mathrm{Te}_{3}$ phase resulting from co-doping $\mathrm{Sb}_{2} \mathrm{Te}_{3}$ films. Our study highlights the importance of simultaneous electrical and optical probing methods to understand the properties of exotic magnetic materials, a building block for dissipationless topological spintronic devices. 


\section{MOKE Instrumentation}

(a)

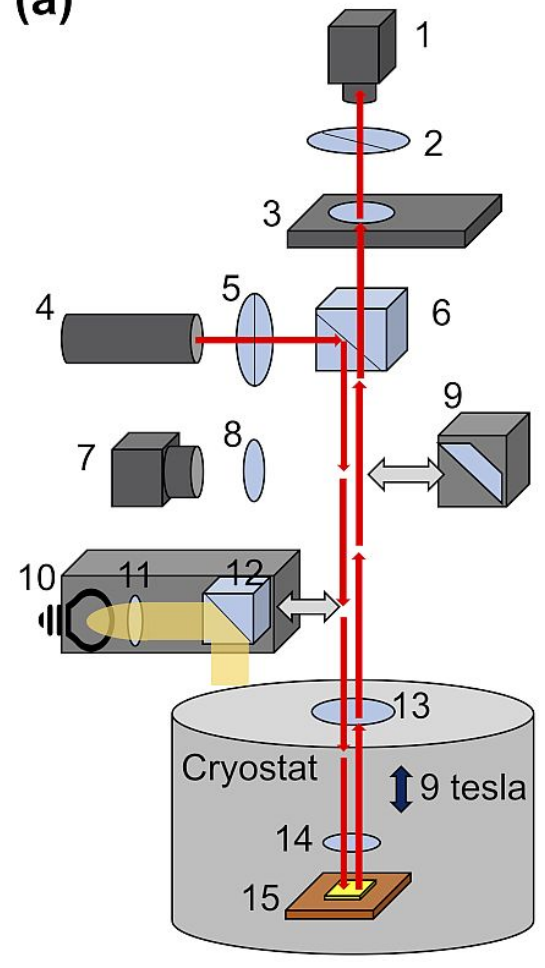

(b)

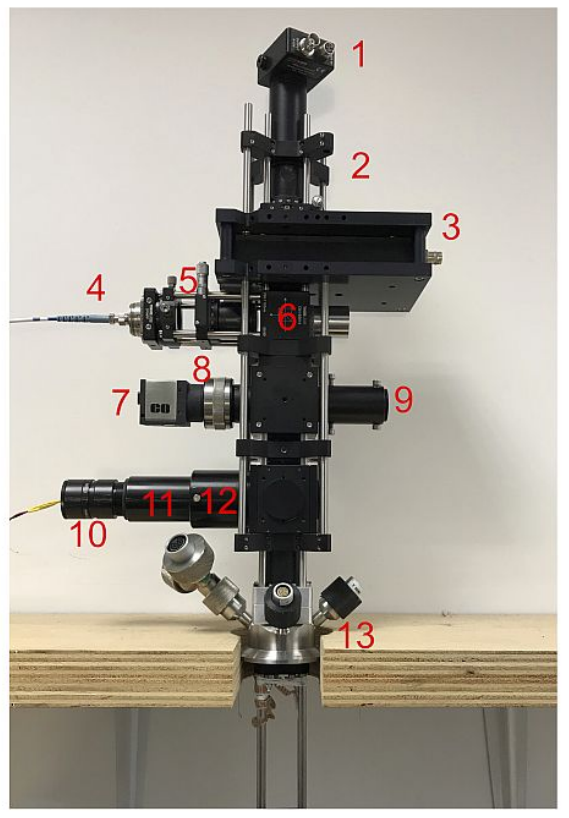

(c)

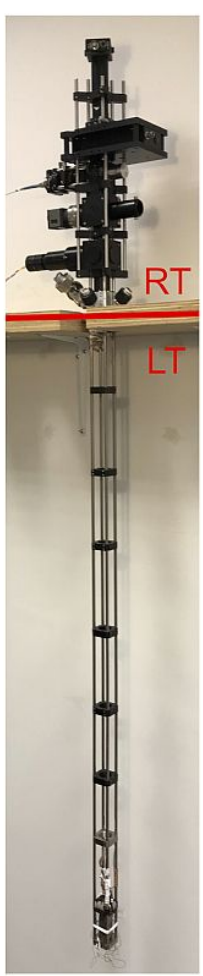

Figure 1. (a) Diagram of the MOKE probe (not to scale) with the optical components as follows: (1) photodetector, (2) photoelastic modulator (PEM), (3) polariser at $45^{\circ}$, (4) fibre-coupled 850-nm laser, (5) polariser at $0^{\circ}$, (6) beamsplitter, (7) camera, (8) imaging lens, (9) $45^{\circ}$ mirror, (10) LED bulb, (11) collimation lens, (12) beamsplitter, (13) cryostat window, (14) objective lens, and (15) sample on piezoelectric stage. (b) Photograph of the room temperature part of the MOKE probe. The numbering of the optical components is identical to panel (a). (c) Photograph of the entire MOKE probe. The height of the room temperature (RT) part is $0.5 \mathrm{~m}$ and that of the low temperature (LT) part $1.2 \mathrm{~m}$.

We constructed a polar MOKE probe where the surface of the sample being examined is perpendicular to both the incident light and the external magnetic field. In this geometry, the rotation angle and the ellipticity change of the reflected light are proportional (first order approximation) to the out-of-plane magnetisation of the material. This MOKE probe is a stand-alone mechanical assembly that can be partly inserted into a helium-4 continuous flow cryostat (Oxford Instruments), which has a base temperature of $1.6 \mathrm{~K}$ and is equipped with a $9 \mathrm{~T}$ superconducting magnet.

Figure 1 shows the schematic diagram (not to scale) and photos of the MOKE probe. The optical head is external to the cryostat, and rigidly mounted on the KF50 cryostat flange. Below this, the insert assembly consists of a truss comprising four hollow Grade-2 titanium tubes, with 6 thermal baffles distributed along the $1.2 \mathrm{~m}$ length adding rigidity. The structure at the end of the truss is machined from solid titanium 
and holds the optical objective and, below it, a three-axis translation system. The stickslip XYZ stage with resistive position readout permits the sample mounted on the top to be placed with precision of about $50 \mathrm{~nm}$. There are 20 electrical contacts wired on the sample holder to support the magneto-transport measurements. The optical system is based on standard commercial components.

Light from a continuous-wave diode laser operated at $850 \mathrm{~nm}$ with stabilised optical output power and temperature is coupled into a polarisation-maintaining (PM) singlemode fibre [labelled as (4) in Figure 1]. This cleans the spatial mode from the laser, and the exiting light is collimated at the entry to the MOKE head to produce a free-space beam with Gaussian transverse profile, which has a beam-waist parameter of about $1 \mathrm{~mm}$. The Rayleigh range of this beam exceeds the path length within the apparatus, so the beam profile is almost constant except where focussed. The collimated laser light then passes through a Glan-Taylor polariser (5) at $0^{\circ}$ to ensure a high degree of controlled linear polarisation. This is deflected by the non-polarising beamsplitter (6) into the main optical axis of the probe. For the MOKE measurement, the light passes through a fused silica window into the cryostat, and down to the objective (14). This is a moulded aspherical lens with a numerical aperture of 0.5 , optimised to produce a spot of less than $2 \mu \mathrm{m}$ in diameter. The light reflected from the sample is re-collimated by the objective lens (14) and exits the cryostat through the same optical path, passing through the cube beamsplitter (6), a photoelastic modulator [PEM, (3)], and another Glan-Taylor polariser (2) that is aligned at $45^{\circ}$ to the initial polarisation. Finally the beam hits a biased Si photodiode, which converts the photocurrent to a usable voltage by a transimpedance amplifier. Initial alignment is greatly facilitated by the inclusion of two removable elements in the MOKE head: a Köhler illuminator (10-12) and camera system focussed on infinity (7-8) fed by an additional beamsplitter (9). This provides an image of the sample with $20 \mu \mathrm{m}$ spatial resolution when these elements are inserted into the main beam path. This function is particularly useful as a pre-positioning before measuring the Kerr response of a specific area of the material.

Some low-temperature Kerr magnetometers use PM fibres, rather than a free-space beam, as the main method of laser transmission [37]. They have been employed, e.g., to study broken TRS in unconventional superconductors [38, 39]. In fibre-coupled MOKE microscopes, longitudinal MOKE is possible even in a constrained sample space, which is an advantage over the free-space approach. However, using the PM fibre scheme may result in blocking the central optical axis and thus real-time optical imaging of the sample, thereby preventing the initial alignment when only magnetic microstructures are to be examined.

The polarisation of the light is modulated by the PEM utilising the photoelastic effect. In the PEM there is a piezoelectric transducer that compresses and stretches a fused silica window at its resonant frequency $(50 \mathrm{kHz})$. This induces birefringence in the window that modulates the transmitted light of a certain wavelength between leftand right-circular polarisation at $50 \mathrm{kHz}$. In our optical geometry, the Kerr rotation 
angle and ellipticity can be derived using the following equations [40, 41]:

$$
\begin{aligned}
\theta_{K} & =\frac{\sqrt{2}}{4 J_{2}(a)} \frac{V_{2 f}}{V_{d c}} \\
\epsilon_{K} & =\frac{\sqrt{2}}{4 J_{1}(a)} \frac{V_{f}}{V_{d c}}
\end{aligned}
$$

where $J_{1}$ and $J_{2}$ are the first and second order Bessel functions of the tunable retardation phase $a$ of the PEM, and $V_{f}, V_{2 f}$ and $V_{d c}$ are the photovoltages at $50 \mathrm{kHz}, 100 \mathrm{kHz}$ and DC, respectively. In this way both the Kerr rotation and ellipticity of the reflected beam can be detected simultaneously. Apart from using the photoelastic effect, another common modulation scheme in a MOKE microscope is the differential detection. In this scheme the reflected beam is split into two cross-polarised beams and collected by a differential photodetector with two balanced photodiodes [4, 15, 16, 42]. Here we use the PEM modulation scheme because it has a better tolerance to misalignment, as the reflected light always stays in the central optical path without splitting.

\section{Two-dimensional magnetic scanning}

This stand-alone MOKE microscope is designed under the principle of confocal laser scanning microscopy, i.e., a focused laser beam is scanned across the whole sample to generate a magnetic image. A perpendicularly magnetised thin film was grown and micropatterned to verify the two-dimensional scanning capability of the MOKE probe at low temperatures. The $[\mathrm{CoFeB} / \mathrm{Pt}]_{n}$ multilayers have outof-plane anisotropy originating from interface exchange coupling [43, 44]. The $\mathrm{Pt}(2) /[\mathrm{CoFeB}(0.55) / \mathrm{Pt}(0.7)]_{4} / \mathrm{CoFeB}(0.55) / \mathrm{Pt}(2) / \mathrm{Ta}(2)$ (thicknesses in nm) multilayer structure was grown on a $\mathrm{Si}$ substrate using magnetron sputtering. After the initial deposition of the Ta buffer layer on the $\mathrm{Si}(100)$ substrate, $\mathrm{Pt}$ and $\mathrm{CoFeB}$ were alternately sputtered from individual targets onto the film. The substrate was kept at room temperature and the chamber pressure was at $7.1 \times 10^{-3}$ mbar throughout the deposition. After growth the sample was taken out and its perpendicular magnetic anisotropy at the ambient conditions was confirmed using a commercial MOKE magnetometer. This multilayer sample was then fabricated into pre-designed structures by standard optical lithography and $\mathrm{Ar}^{+}$dry etching techniques. First, negative photoresist was spincoated onto the film, followed by patterning and exposure using a MicroWriter (Durham Magneto Optics). Afterwards, the pattern was etched using $\mathrm{Ar}^{+}$milling and a standard lift-off process. Finally the wafer was loaded into the low-temperature MOKE probe and cooled to $6 \mathrm{~K}$. A two-dimensional magnetisation scan was recorded using LabView.

Figure 2(a) shows a two-dimensional MOKE scan at $6 \mathrm{~K}$ of the micro-pattern, comprising the word 'CAMBRIDGE' formed in capitals with $5 \mu \mathrm{m}$-wide strokes of a magnetic $[\mathrm{CoFeB} / \mathrm{Pt}]_{n}$ multilayer structure, on a bare $\mathrm{Si}(100)$ substrate. An out-of-plane magnetic field of $+0.5 \mathrm{~T}$ was first applied and then removed so that the magnetisation of the multilayer stays at the positive remanence. We scanned the laser across an area 


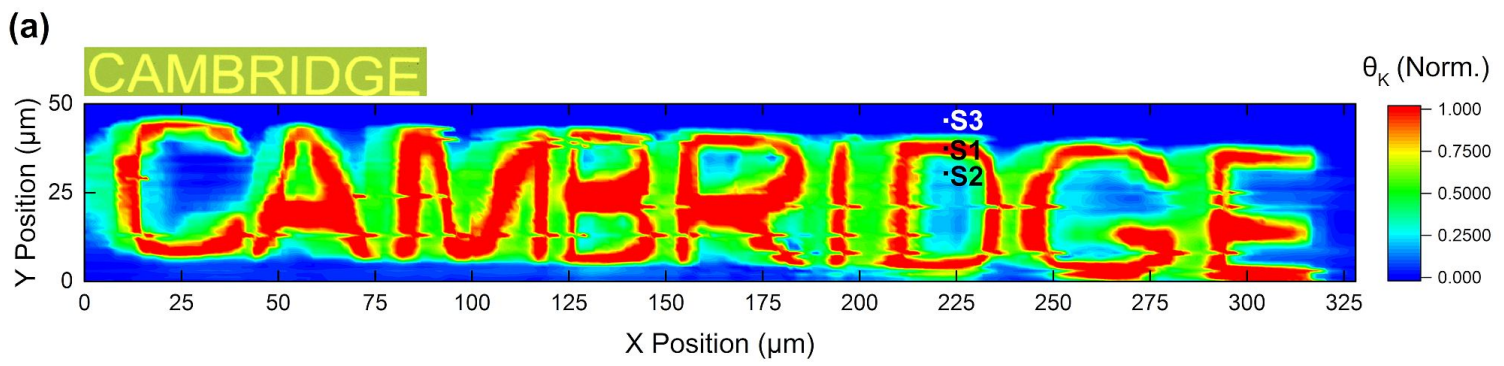

(b)

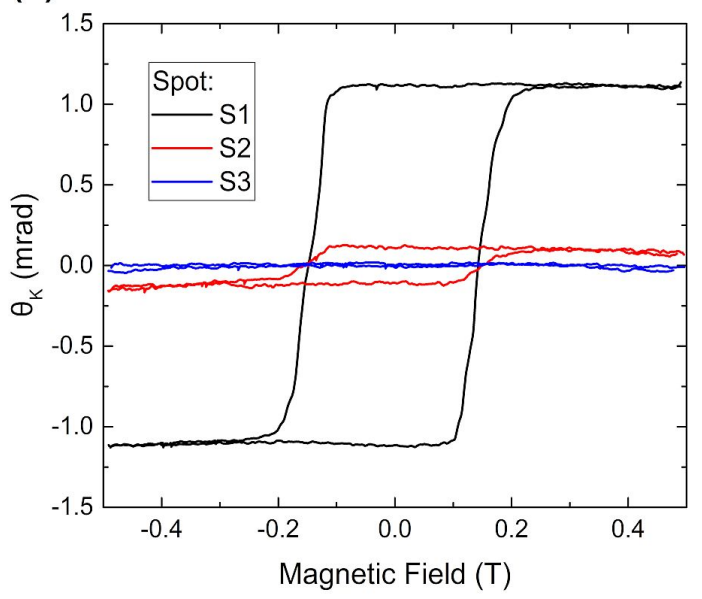

(c)

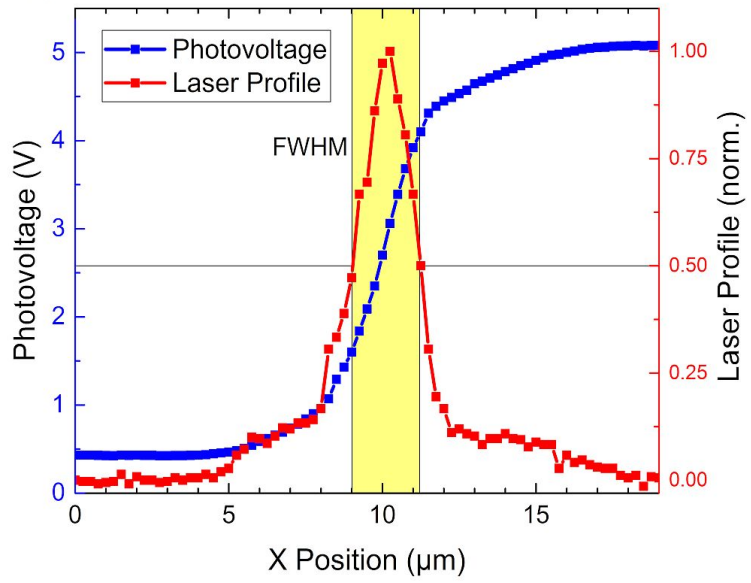

Figure 2. (a) Two-dimensional MOKE scan of the ferromagnetic pattern 'CAMBRIDGE' at $6 \mathrm{~K}$, fabricated from a $[\mathrm{CoFeB} / \mathrm{Pt}]_{n}$ multilayer film with out-ofplane anisotropy. The letter stroke width is $5 \mu \mathrm{m}$ and the scanning step size is $1 \mu \mathrm{m}$. An optical micrograph of the same area is shown at the top left corner. (b) Magnetic hysteresis loops of three different spots on or next to the 'CAMBRIDGE' pattern. (c) The photovoltage recorded (in blue) and its derivative, i.e., the laser profile (in red), when the laser scans across a knife edge. The yellow shaded area represents full width at half maximum (FWHM) of the laser spot.

of $350 \mu \mathrm{m} \times 50 \mu \mathrm{m}$ with $1 \mu \mathrm{m}$ step size and recorded both the AC Kerr angle and DC reflectance. On this map every character can be clearly recognised with moderately sharp edge. The gradual magnetisation change at the edges of those letters is perhaps due to the resolution limit of the focused laser spot. The small horizontal lines in the map arise from position readout errors. Another Kerr scan at remanence, but after applying a $-0.5 \mathrm{~T}$ preset field, revealed an identical magnetisation distribution with opposite rotation angles (not shown here), proving that the Kerr signal does originate from the $[\mathrm{CoFeB} / \mathrm{Pt}]_{n}$ structure. As a reference, an image of the text taken from an optical microscope is displayed at the top left corner of the Kerr map. To examine further the magnetisation at individual $x-y$ coordinates, we measured the hysteresis loops when focusing the laser at Spot S1 on letter D, S2 in the hollow part of letter D, and S3 off the letter. The results are shown in Figure 2(b) after subtracting a linear background arising from Faraday rotation in the objective lens. Spot S1 has a square-shaped loop indicating the magnetic easy axis is perpendicular to the film. The hysteresis loop at 
Spot S2 shows the same coercivity as S1 but the magnetisation is smaller. This small but existing magnetic response is perhaps due to a fraction of the light reflected from the magnetic letters nearby. In comparison, no magnetic response was found on Spot S3 which is away from the letters. Close examination of the hysteresis loops shows the noise level is less than $10 \mu \mathrm{rad}$ at $6 \mathrm{~K}$. Finally, the focused laser was scanned across a knife edge with a step size of $0.25 \mu \mathrm{m}$. The intensity of the reflected light and its differentiation at each step are illustrated in Figure 2(c). The laser profile is close to a Gaussian with $2.2 \mu \mathrm{m}$ full width at half maximum (FWHM, shaded in yellow in the figure). The minimum spot size of a laser is given approximately by the beam waist

$w_{0}=\frac{\lambda}{\pi N A}$, where $w_{0}$ is the radius at the point where intensity falls to $\frac{1}{e^{2}}$ of the maximum value, and $N A$ is the numerical aperture. This gives a value slightly smaller than that determined experimentally.

Apart from the laser scanning microscope shown above, many Kerr microscope designs rely on wide-field microscopes, which use a broadband light source to illuminate the whole specimen, and therefore provide a real-time polarised image of the sample $[45,46]$. Compared to the scanning microscopes, wide-field Kerr microscopes have the advantage of direct visualisation of magnetic structures at a fast acquisition rate [47]. Because of this, they have been extensively employed for magnetic domain observations [48]. Other examples of wide-field MOKE applications include resolving individual vortices in type-II superconductors and skyrmion bubbles in magnetic trilayer films $[46,49,50]$. We chose to construct our stand-alone MOKE probe using the scanning laser scheme because it is more tailored for high-sensitivity magnetometry, achieving quantitative Kerr results that complement electrical transport results on MTIs and other magnetic materials. It is possible to further develop a Kerr microscope with combined scanning and wide-field configurations to utilise the virtues of both, and a good example can be found in Ref. [47].

\section{Investigation of the magnetic topological insulators $(\mathrm{Cr}, \mathrm{V}): \mathrm{Sb}_{2} \mathrm{Te}_{3}$ and $\mathrm{Cr}: \mathrm{Sb}_{2} \mathrm{Te}_{3}$}

The (Cr, V):Sb $\mathrm{Se}_{3}$ and $\mathrm{Cr}: \mathrm{Sb}_{2} \mathrm{Te}_{3}$ TI thin films were grown on c-plane sapphire substrates by molecular beam epitaxial (MBE) co-deposition with a base chamber pressure of $1 \times 10^{-10}$ mbar [33, 36, 51]. Sb and Te elements were evaporated from $6 \mathrm{~N}$ purity sources in standard effusion cells, while $\mathrm{Cr}$ and $\mathrm{V}$ elements were from $4 \mathrm{~N}$ purity sources in high temperature cells. All beam fluxes were measured in situ by a beam flux monitor. The co-doped $(\mathrm{Cr}, \mathrm{V}): \mathrm{Sb}_{2} \mathrm{Te}_{3}$ sample was grown in a overpressure of $\mathrm{Te}$, where the flux ratio $(\mathrm{V}+\mathrm{Cr}+\mathrm{Sb}): \mathrm{Te}=10: 1$ was maintained, which reduces $\mathrm{Te}$ vacancies and anti-site defects common in the $\mathrm{Sb}_{2} \mathrm{Te}_{3}$ system [52]. In the first step of the growth a 5 -nm-thick $\mathrm{Sb}_{2} \mathrm{Te}_{3}$ layer was deposited on the sapphire substrate at 200 ${ }^{\circ} \mathrm{C}$ as a buffer layer, then annealed at $250{ }^{\circ} \mathrm{C}$ with $\mathrm{Te}$ flux on. In the next step a 60 nm-thick (Cr, V): $\mathrm{Sb}_{2} \mathrm{Te}_{3}$ was deposited at $250{ }^{\circ} \mathrm{C}$, with a flux ratio $\mathrm{V}: \mathrm{Cr}=1: 5$. We also grew a single-doped $\mathrm{Cr}: \mathrm{Sb}_{2} \mathrm{Te}_{3}$ thin film with identical $\mathrm{Cr}$ concentration using the same 
procedure but without $\mathrm{V}$ dopant. The nominal composition ratios of the two samples are $\mathrm{Cr}_{0.41} \mathrm{~V}_{0.04} \mathrm{Sb}_{1.65} \mathrm{Te}_{3}$ and $\mathrm{Cr}_{0.41} \mathrm{Sb}_{1.69} \mathrm{Te}_{3}$. Both films show streaky patterns by in situ reflection high energy electron diffraction throughout the deposition (not shown here), indicating a smooth two-dimensional growth mode. The structural property of the grown films was characterised ex situ by x-ray diffraction (XRD) in a Bruker D8 diffractometer.

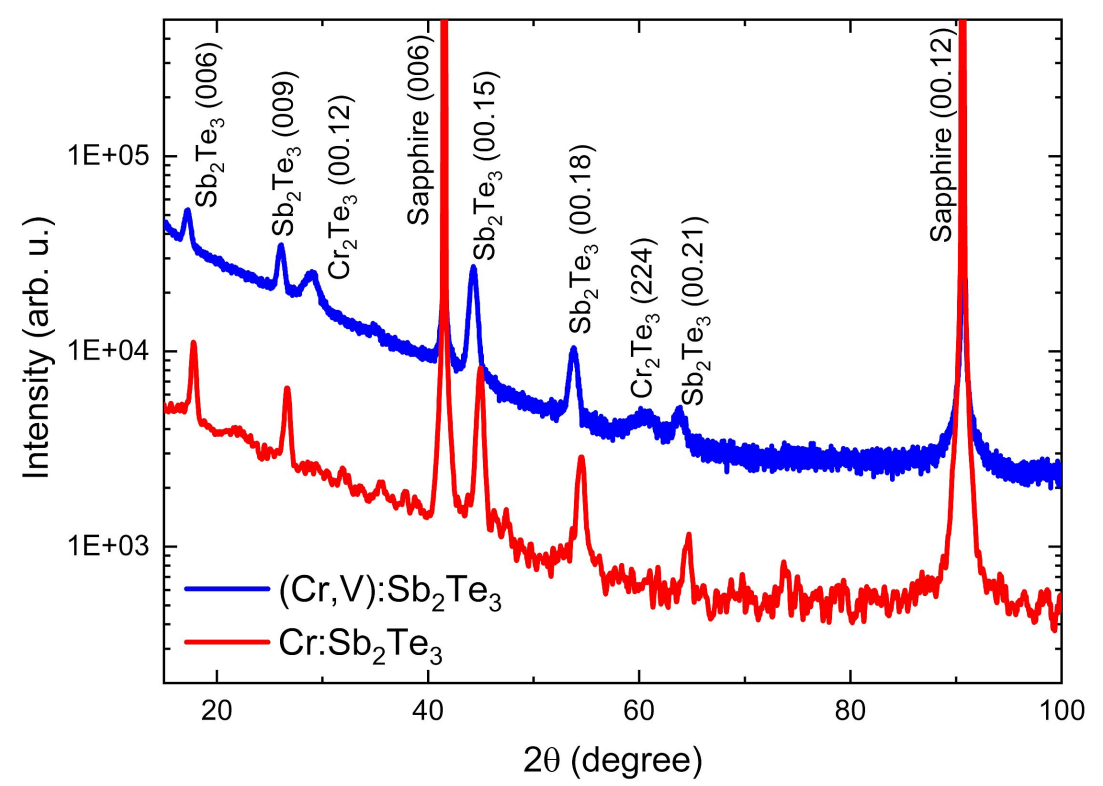

Figure 3. XRD spectra of $(\mathrm{Cr}, \mathrm{V}): \mathrm{Sb}_{2} \mathrm{Te}_{3}$ (in blue) and $\mathrm{Cr}_{\mathrm{Sb}} \mathrm{Sb}_{2} \mathrm{Te}_{3}$ (in red) MTI thin films.

The XRD spectra of the (Cr,V): $\mathrm{Sb}_{2} \mathrm{Te}_{3}$ (in blue) and $\mathrm{Cr}: \mathrm{Sb}_{2} \mathrm{Te}_{3}$ (in red) thin films are illustrated in Figure 3. The $\left(\begin{array}{l}0 \\ 0\end{array}\right)$ peaks of $\mathrm{Sb}_{2} \mathrm{Te}_{3}$ in the single-doped $\mathrm{Cr}: \mathrm{Sb}_{2} \mathrm{Te}_{3}$ film indicate the presence of a well-ordered crystal structure, with the $c$-axis parallel to the surface normal. No secondary phases such as $\mathrm{SbTe}, \mathrm{SbTe}_{2}$ or $\mathrm{Sb}_{2} \mathrm{Te}_{5}$ can be found in this spectrum. The result indicates that the $\mathrm{Sb}_{2} \mathrm{Te}_{3}$ structure remains intact upon moderate $\mathrm{Cr}$ doping. The $(\mathrm{Cr}, \mathrm{V}): \mathrm{Sb}_{2} \mathrm{Te}_{3}$ sample, on the other hand, shows the presence of another phase, $\mathrm{Cr}_{2} \mathrm{Te}_{3}$, in addition to $\mathrm{Sb}_{2} \mathrm{Te}_{3}$. We did not find diffraction peaks related to any $\mathrm{V}$-Te compound, such as VTe or $\mathrm{V}_{3} \mathrm{Te}_{4}$. Our observation of $\mathrm{Cr}_{2} \mathrm{Te}_{3}$ is consistent with the previous finding that vanadium acts as a surfactant preventing $\mathrm{Cr}$ from substituting $\mathrm{Sb}$ in the co-doped (Cr,V): $\mathrm{Sb}_{2} \mathrm{Te}_{3}$ structure, forcing it to form $\mathrm{Cr}_{2} \mathrm{Te}_{3}$, which has similar crystallographic parameters to $\mathrm{Sb}_{2} \mathrm{Te}_{3}[33,53]$.

For the electrical transport and MOKE measurements, the (Cr, V): $\mathrm{Sb}_{2} \mathrm{Te}_{3}$ and $\mathrm{Cr}: \mathrm{Sb}_{2} \mathrm{Te}_{3}$ thin films were fabricated into Hall bars of dimensions $1400 \mu \mathrm{m} \times 150 \mu \mathrm{m}$ using optical lithography and $\mathrm{Ar}^{+}$ion milling in a similar way to the $[\mathrm{CoFeB} / \mathrm{Pt}]_{n}$ film. This was followed by the thermal evaporation of 20/80-nm-thick Ti/Au and lift-off to form ten Ohmic contacts. The micro-devices were then glued and wire-bonded to a 20-pin leadless chip carrier and placed onto the sample holder of the MOKE probe before loading into the cryostat. We scanned the light reflectivity across the devices and 
mapped out their respective $x-y$ coordinates. In order to verify the planar magnetisation uniformity of both samples, we moved the laser spot to various places on the Hall bars. Variation of Kerr response at different locations was not significant. Finally we focused the laser to the geometrical centre of the Hall bars throughout the rest of the experiments. We measured electrical transport properties of the devices simultaneously with optical probing, using a standard four-terminal AC lock-in scheme with an input current of $1 \mu \mathrm{A}$ at a frequency of $77 \mathrm{~Hz}$.

(a)

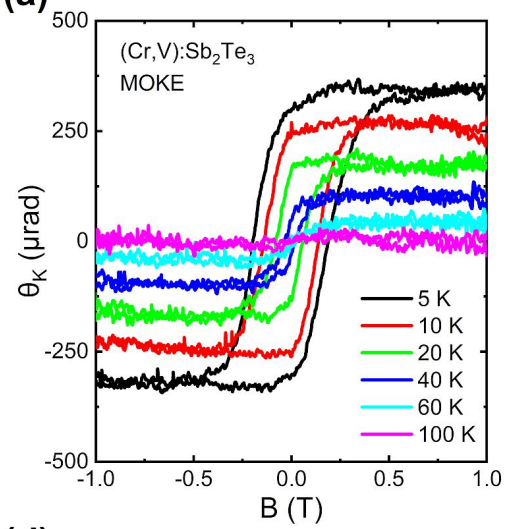

(d)

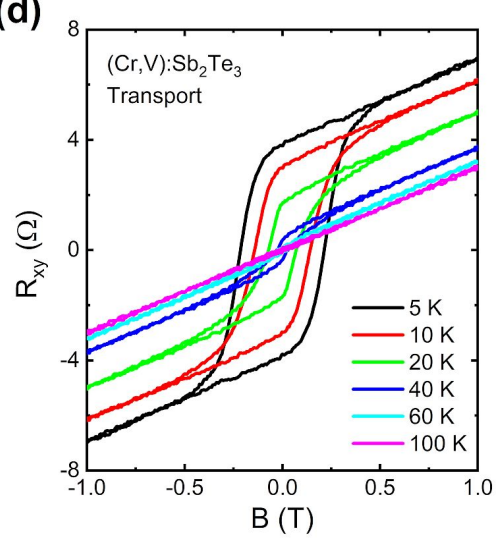

(b)

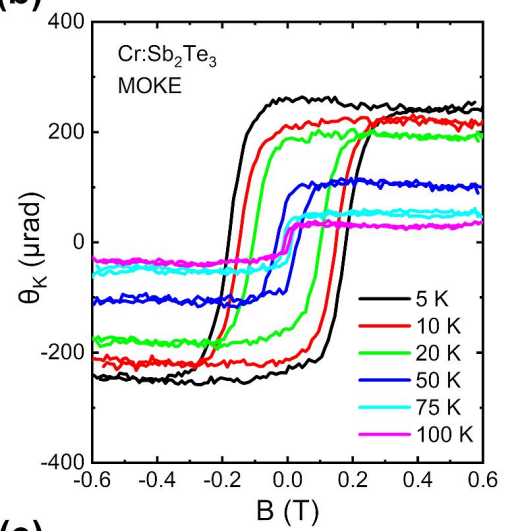

(e)

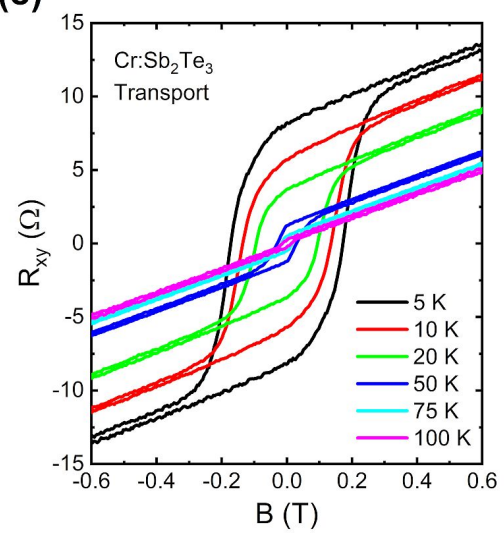

(c)

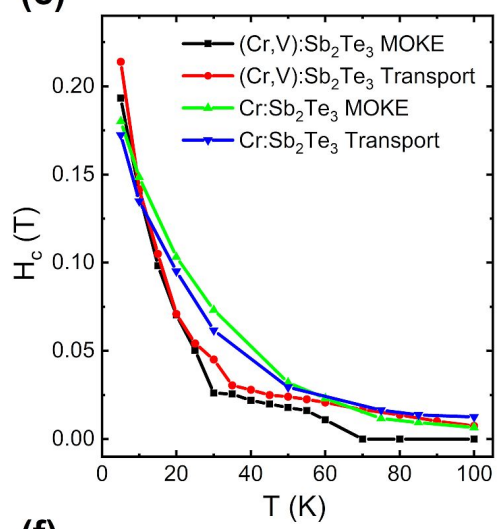

(f)

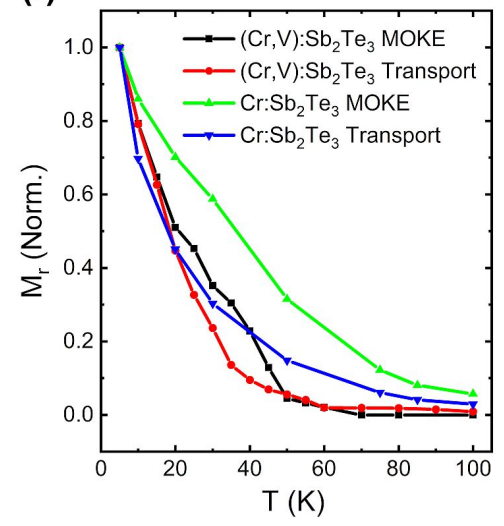

Figure 4. Temperature-dependent magnetic hysteresis loops of (a) (Cr, V): $\mathrm{Sb}_{2} \mathrm{Te}_{3}$ and (b) $\mathrm{Cr}_{\mathrm{Sb}} \mathrm{Te}_{3}$ films obtained from MOKE measurements. (d,e) Corresponding hysteresis loops obtained from AHE electrical transport measurements. (c,f) Temperature-dependent coercivity and saturation magnetisation values extracted from MOKE and transport measurements for the two films.

Figures 4(a,d) illustrate the magnetic hysteresis loops of the $(\mathrm{Cr}, \mathrm{V}): \mathrm{Sb}_{2} \mathrm{Te}_{3}$ film at different temperatures ranging from $5 \mathrm{~K}$ to $100 \mathrm{~K}$ using combined optical and electrical techniques. The results obtained display the AHE effect, showing good consistency between the two measurement schemes. Figures 4(b,e) show corresponding results for the single-doped $\mathrm{Cr}_{\mathrm{Sb}} \mathrm{Sb}_{2} \mathrm{Te}_{3}$ film. From the shape of the loops we can conclude that both materials establish a strong ferromagnetic order perpendicular to the film plane, with the magnitude of the remanence $\left(M_{r}\right)$ at zero field close to the spontaneous magnetisation $\left(M_{s}\right)$ at high fields. To compare better the results between the two samples, and also 
between the two measurement schemes, we plotted the coercivity $\left(H_{c}\right)$ and remanent magnetisation $\left(M_{r}\right)$ extracted from these four graphs in Figures $4(\mathrm{c}, \mathrm{f})$. The electricallyand optically-obtained coercive fields of both samples match quantitatively across the whole temperature range. The remanent magnetisation, on the other hand, displays a concave-shaped trend in both materials following the temperature increment, consistent with observations in other magnetic-doped $(\mathrm{Bi}, \mathrm{Sb})_{2}(\mathrm{Te}, \mathrm{Se})_{3}$ TI systems $[32,36,51]$. In both samples, $M_{r}$ sees a steeper decrease in the Hall resistance compared to the Kerr angle. Surface sensitivity of the Kerr measurement may account for the difference in $M_{r}$ compared to transport measurement, which is bulk sensitive. The good consistency between the optically and electrically probed $H_{c}$ and $M_{r}$ may be relevant in the context of the recent discovery of the THE in TI heterostructures [34, 35], where the value of the THE can be obtained when the transport results are directly subtracted from the MOKE readings $[18,21]$.
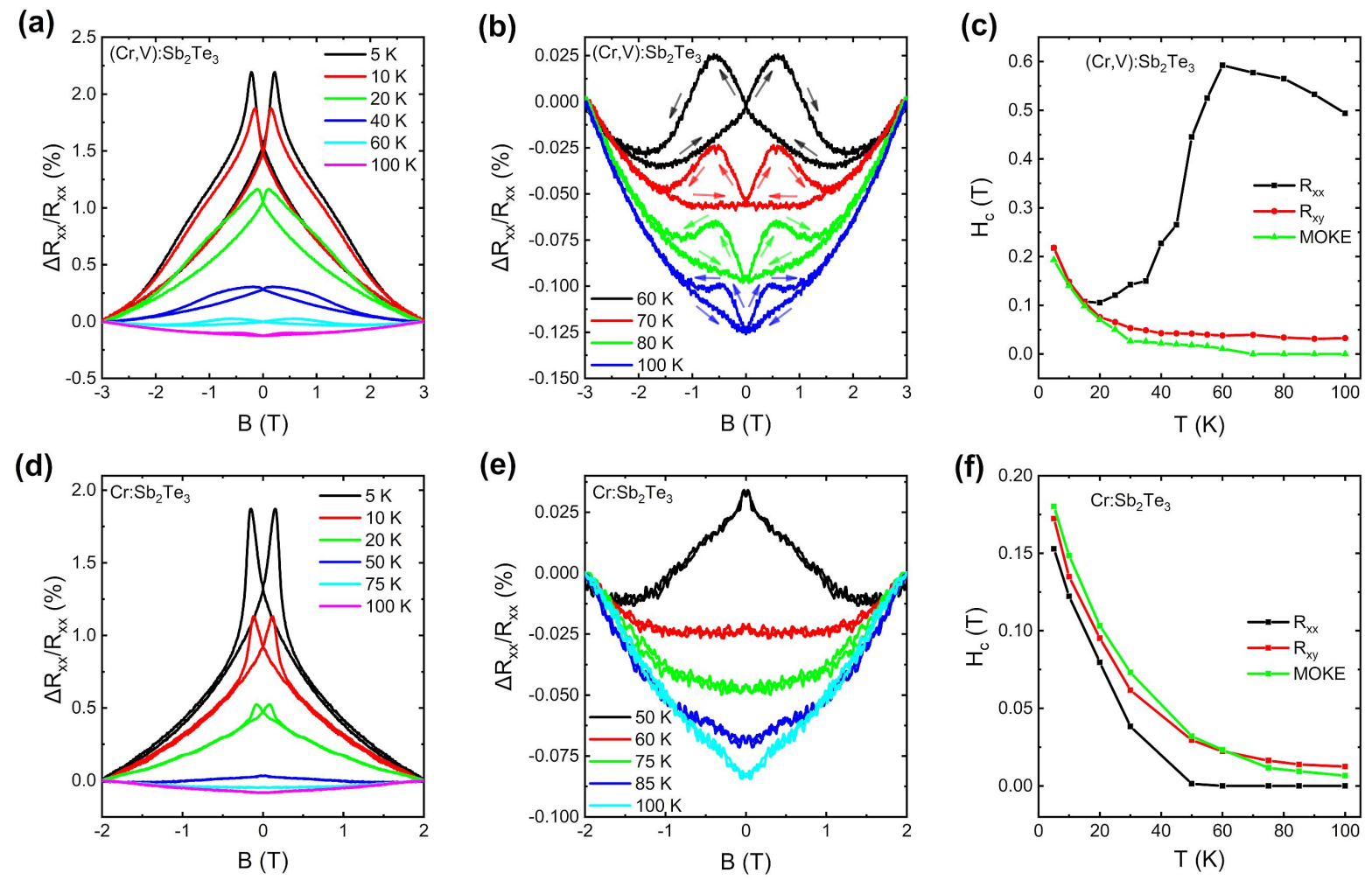

Figure 5. (a) Temperature-dependent $\mathrm{MR}$ of the $(\mathrm{Cr}, \mathrm{V}): \mathrm{Sb}_{2} \mathrm{Te}_{3}$ film from $5 \mathrm{~K}$ to 100 $\mathrm{K}$. (b) Close-up of the MR above $60 \mathrm{~K}$, with arrows indicating the direction of the magnetic field sweeps. (c) Comparison between the magnetic fields obtained from the resistance peaks in the MR measurements $\left(R_{x x}\right)$, and the coercive fields obtained from MOKE and $R_{x y}$. (d,e,f) Corresponding results for the $\mathrm{Cr}: \mathrm{Sb}_{2} \mathrm{Te}_{3}$ film.

The results of magnetoresistance $\left(\mathrm{MR}, R_{x x}\right)$ measurements of the $(\mathrm{Cr}, \mathrm{V}): \mathrm{Sb}_{2} \mathrm{Te}_{3}$ and $\mathrm{Cr}: \mathrm{Sb}_{2} \mathrm{Te}_{3}$ films at various temperatures are summarised in Figure 5. Figures 5(a-c) show the MR results of the of the co-doped (Cr,V): $\mathrm{Sb}_{2} \mathrm{Te}_{3}$ sample, and $5(\mathrm{~d}-\mathrm{f})$ show the results of the single-doped $\mathrm{Cr}: \mathrm{Sb}_{2} \mathrm{Te}_{3}$ film. For presentation purposes, the field- 
dependent MR ratio $\Delta R_{x x} / R_{x x}$ is defined as $\left(R_{x x}(B)-R_{x x}\left(B_{\max }\right)\right) / R_{x x}\left(B_{\max }\right)$. The MR curves of $(\mathrm{Cr}, \mathrm{V}): \mathrm{Sb}_{2} \mathrm{Te}_{3}$ demonstrate the anisotropic magnetoresistance (AMR) below $60 \mathrm{~K}$, shown in Figure 5(a), where the resistance reaches its maximum value during magnetisation reversal. Surprisingly, in the co-doped film above $70 \mathrm{~K}$, the AMR feature still persists with flattened peaks, and a dip emerges in the resistance curve at zero magnetic field, as is shown in Figure 5(b). In a pristine TI material, time-reversal backscattering of electrons is suppressed due to spin-momentum locked surface states, resulting in the weak-antilocalisation (WAL) effect, where the sample resistance reaches a minimum at zero field $[54,55]$. WAL is a strong indication of the existence of TI surface states [56-59]. Above $70 \mathrm{~K}$, the WAL contribution becomes progressively dominant with increasing temperature compared to AMR. This unusual response may arise from a joint effect of WAL from the $\mathrm{Sb}_{2} \mathrm{Te}_{3}$ phase and $\mathrm{AMR}$ from the $\mathrm{Cr}_{2} \mathrm{Te}_{3}$ phase - the latter being a ferromagnetic compound with a Curie temperature of over $100 \mathrm{~K}$ [53]. These results are consistent with the presence of the $\mathrm{Cr}_{2} \mathrm{Te}_{3}$ phase. Figure 5(c) shows the difference of $H_{c}$ extracted from $R_{x x}, R_{x y}$ and the MOKE measurements. From $20 \mathrm{~K}$ to $60 \mathrm{~K}$, the distance of the MR peaks corresponding to the coercive field for $(\mathrm{Cr}, \mathrm{V}): \mathrm{Sb}_{2} \mathrm{Te}_{3}$ (low $T_{\mathrm{C}}$ ) show an increment. This is due to the gradual overshadowing of the $\mathrm{Cr}_{2} \mathrm{Te}_{3}$ (high $T_{\mathrm{C}}$ ) ferromagnetic phase. On the contrary, the single-doped sample shows a clear transition from AMR to WAL above $50 \mathrm{~K}$ with matching coercivities among $R_{x x}, R_{x y}$ and MOKE, as shown in Figure $5(\mathrm{~d}, \mathrm{e}, \mathrm{f})$. This further proves that the inconsistency between the coercivity results in $(\mathrm{Cr}, \mathrm{V}): \mathrm{Sb}_{2} \mathrm{Te}_{3}$ come from the ferromagnetic $\mathrm{Cr}_{2} \mathrm{Te}_{3}$ secondary phase, which is not presented in the single-doped film.

High-field superconducting magnets, which were used for the MTI samples, are a standard feature of transport cryostats. However, this high-field capability is rarely found in optical cryostats, except in a few commercial magneto-optical measurements stations. It is therefore standard practice to carry out low-field magneto-optic measurements and high-field transport measurements in separate cryostats [60]. The stand-alone MOKE probe provides a cost-effective solution for the exploration of the high-field magneto-optic properties of MTIs and other magnetic systems. On the other hand, optical cryostats provide better flexibility and are able to accommodate various measurement geometries, such as polar, longitudinal and transverse MOKE, and outperform the stand-alone probe in terms of noise reduction since most of optical components are mounted on a vibration-damped optical table [17].

\section{Summary and Conclusion}

We presented the design and demonstrated the performance of a low-temperature, focused polar MOKE microscope for use with a base temperature of $1.6 \mathrm{~K}$, which is equipped with a $9 \mathrm{~T}$ superconducting magnet. Instead of using a cold-finger with a transparent window, we integrated the Kerr magnetometer into a conventional cryostat, demonstrating a practical approach to set up low-temperature microscopy instrumentation for non-optical cryostat users. The laser travels as a free-space 
beam in the cryostat, and the polarisation modulation at $50 \mathrm{kHz}$ is achieved by employing a PEM. We performed a two-dimensional Kerr scan at $6 \mathrm{~K}$ on ferromagnetic $[\mathrm{CoFeB} / \mathrm{Pt}]_{n}$ multilayer microstructures with perpendicular interfacial anisotropy. A clearly resolved word pattern with $5 \mu \mathrm{m}$ stroke width confirms the capability of microscale magnetisation mapping. Another scan across a knife edge reveals a focal spot size of $2.2 \mu \mathrm{m}$ FWHM, close to the predicted diffraction limit. We further utilised the MOKE probe to carry out a simultaneous study of magneto-transport and magneto-optic effects on $\mathrm{Cr}: \mathrm{Sb}_{2} \mathrm{Te}_{3}$ and co-doped (Cr,V): $\mathrm{Sb}_{2} \mathrm{Te}_{3}$ MTI thin films. Both materials show longrange ferromagnetic order at low temperature with the magnetic easy axis pointing outof-plane. The consistency of the results obtained using the two measurement schemes is demonstrated for these MTI samples, with both the coercivity $\left(H_{c}\right)$ and magnetisation $\left(M_{r}\right)$ matching well. The appearance of a secondary $\mathrm{Cr}_{2} \mathrm{Te}_{3}$ phase in the co-doped sample is echoed by an unexpected MR response, as the coexistence of the two phases resulted in a combination of AMR and WAL effects above $70 \mathrm{~K}$. The comparison of coercivities among the MOKE, $R_{x x}$, and $R_{x y}$ results further confirms the presence of a secondary ferromagnetic phase. Our study highlights the advantages of employing both magneto-optic and magneto-transport techniques to study MTI systems, providing a pathway to understand exotic topological materials for energy-dissipationless spintronic applications. Considering the large community of electrical transport physics and the emerging trend of combining electrical and optical studies on solid state systems, we believe our demonstration will appeal to transport scientists who wish to independently carry out magneto-optic research on MTI and other magnetic materials.

\section{Acknowledgements}

A.S. acknowledges financial support from the SGPC Cambridge Commonwealth Trust (CCT). J. Llandro and C.H.W.B. are grateful for financial support from EPSRC (Grant No. EP/J00412X/1). T.H. acknowledges financial support from the John Fell Oxford University Press Research Fund. L.B.D. acknowledges financial support from EPSRC and the Science and Technology Facilities Council (UK). M.R.S. acknowledges support from the Cambridge Commonwealth, European and International Trust and the EPSRC Cambridge NanoDTC (Grant No. EP/L015978/1).

\section{References}

[1] Argyres P N 1955 Phys. Rev. 97 334-345

[2] Qiu Z Q and Bader S D 1999 J. Magn. Magn. Mater. 200 664-678

[3] Cormier M, Ferré J, Mougin A, Cromières J P and Klein V 2008 Rev. Sci. Instrum. 79033706

[4] Flajšman L, Urbánek M, Křřžáková V, Vaňatka M, Turčan I and Šikola T 2016 Rev. Sci. Instrum. 87053704 
[5] Allwood D A, Xiong G, Cooke M D and Cowburn R P 2003 J. Phys. D: Appl. Phys. 36 2175-2182

[6] Nikulina E, Idigoras O, Vavassori P, Chuvilin A and Berger A 2012 Applied Physics Letters 100142401

[7] Y K Kato, R C Myers, A C Gossard and D D Awschalom 2004 Science 306 19101913

[8] Stamm C, Murer C, Berritta M, Feng J, Gabureac M, Oppeneer P M and Gambardella P 2017 Phys. Rev. Lett. 119087203

[9] Bottegoni F, Zucchetti C, Dal Conte S, Frigerio J, Carpene E, Vergnaud C, Jamet M, Isella G, Ciccacci F, Cerullo G and Finazzi M 2017 Phys. Rev. Lett. 118167402

[10] Crooker S A, Furis M, Lou X, Adelmann C, Smith D L, Palmstrøm C J and Crowell P A 2005 Science 309 2191-2195

[11] Kotissek P, Bailleul M, Sperl M, Spitzer A, Schuh D, Wegscheider W, Back C H and Bayreuther G 2007 Nat. Phys. 3 872-877

[12] Gong C, Li L, Li Z, Ji H, Stern A, Xia Y, Cao T, Bao W, Wang C, Wang Y, Qiu Z Q, Cava R J, Louie S G, Xia J and Zhang X 2017 Nature 546 265-269

[13] Huang B, Clark G, Navarro-Moratalla E, Klein D R, Cheng R, Seyler K L, Zhong D, Schmidgall E, McGuire M A, Cobden D H, Yao W, Xiao D, Jarillo-Herrero P and Xu X 2017 Nature 546 270-273

[14] Fache T, Tarazona H S, Liu J, L'Vova G, Applegate M J, Rojas-Sanchez J C, Petit-Watelot S, Landauro C V, Quispe-Marcatoma J, Morgunov R, Barnes C H and Mangin S 2018 Phys. Rev. B 98064410

[15] Jiménez E, Mikuszeit N, Cuñado J L F, Perna P, Pedrosa J, Maccariello D, Rodrigo C, Niño M A, Bollero A, Camarero J and Miranda R 2014 Rev. Sci. Instrum. 85 053904

[16] Rizo P, Pugžlys A, Liu J, Reuter D, Wieck A, van der Wal C and van Loosdrecht P 2008 Rev. Sci. Instrum. 79123904

[17] Nwokoye C A, Bennett L H, Della Torre E, Siddique A, Narducci F A, Ghahremani M and Khattak K S 2015 Int. J. Nanoparticles Nanotech. 11

[18] Matsuno J, Ogawa N, Yasuda K, Kagawa F, Koshibae W, Nagaosa N, Tokura Y and Kawasaki M 2016 Sci. Adv. 21600304

[19] Xing W, Chen Y, Odenthal P M, Zhang X, Yuan W, Su T, Song Q, Wang T, Zhong J, Jia S et al. 2017 2D Materials 4024009

[20] Fikáček J, Heczko O, Kopeckỳ V, Kaštil J and Honolka J 2018 J. Magn. Magn, Mater. 452 373-379

[21] Ohuchi Y, Matsuno J, Ogawa N, Kozuka Y, Uchida M, Tokura Y and Kawasaki M 2018 Nat. Commun. 9213

[22] Qi X L and Zhang S C 2011 Rev. Mod. Phys. 83 1057-1110 
[23] Yang Q I, Dolev M, Zhang L, Zhao J, Fried A D, Schemm E, Liu M, Palevski A, Marshall A F, Risbud S H and Kapitulnik A 2013 Phys. Rev. B 88081407

[24] Lang M, Montazeri M, Onbasli M C, Kou X, Fan Y, Upadhyaya P, Yao K, Liu F, Jiang Y, Jiang W, Wong K L, Yu G, Tang J, Nie T, He L, Schwartz R N, Wang Y, Ross C A and Wang K L 2014 Nano Lett. 14 3459-3465

[25] Yeats A L, Mintun P J, Pan Y, Richardella A, Buckley B B, Samarth N and Awschalom D D 2017 Proc. Natl. Acad. Sci. U.S.A. 114 10379-10383

[26] Moore J E 2010 Nature 464 194-198

[27] Hasan M Z and Kane C L 2010 Rev. Mod. Phys. 82 3045-3067

[28] Liu Q, Liu C X, Xu C, Qi X L and Zhang S C 2009 Phys. Rev. Lett. 102156603

[29] Chen Y L, Analytis J G, Liu Z K, Igarashi K, Qi X L, Mo S K, Moore R G, Lu D H, Hashimoto M, Sasagawa T, Zhang S C, Fisher I R, Hussain Z and Shen Z X 2010 Science 329 659-663

[30] Chang C Z, Zhang J, Feng X, Shen J, Zhang Z, Guo M, Li K, Ou Y, Wei P, Wang L L, Ji Z Q, Feng Y, Ji S, Chen X, Jia J, Dai X, Fang Z, Zhang S C, He K, Wang Y, Lu L, Ma X C and Xue Q K 2013 Science 340 167-170

[31] Chang C Z, Zhao W, Kim D Y, Zhang H, Assaf B A, Heiman D, Zhang S C, Liu C, Chan M H and Moodera J S 2015 Nat. Mater. 14 473-477

[32] Ou Y, Liu C, Jiang G, Feng Y, Zhao D, Wu W, Wang X X, Li W, Song C, Wang L L, Wang W, Wu W, Wang Y, He K, Ma X C and Xue Q K 2018 Adv. Mater. 30 1703062

[33] Duffy L B, Figueroa A I, van der Laan G and Hesjedal T 2017 Phys. Rev. Materials 1064409

[34] Yasuda K, Wakatsuki R, Morimoto T, Yoshimi R, Tsukazaki A, Takahashi K S, Ezawa M, Kawasaki M, Nagaosa N and Tokura Y 2016 Nat. Phys. 12 555-559

[35] He Q L, Yin G, Grutter A J, Pan L, Che X, Yu G, Gilbert D A, Disseler S M, Liu Y, Shafer P, Zhang B, Wu Y, Kirby B J, Arenholz E, Lake R K, Han X and Wang K L 2018 Nat. Commun. 92767

[36] Singh A, Kamboj V S, Liu J, Llandro J, Duffy L B, Senanayak S P, Beere H E, Ionescu A, Ritchie D A, Hesjedal T and Barnes C H 2018 Sci. Rep. 817024

[37] Kapitulnik A, Xia J, Schemm E and Palevski A 2009 New Journal of Physics 11 055060

[38] Xia J, Maeno Y, Beyersdorf P T, Fejer M and Kapitulnik A 2006 Phys. Rev. Lett. 97167002

[39] Xia J, Schemm E, Deutscher G, Kivelson S, Bonn D, Hardy W, Liang R, Siemons W, Koster G, Fejer M et al. 2008 Phys. Rev. Lett. 100127002

[40] Sato K 1981 Jap. J. Appl. Phys. 20 2403-2409

[41] Polisetty S, Scheffler J, Sahoo S, Wang Y, Mukherjee T, He X and Binek C 2008 Rev. Sci. Instrum. 79055107 
[42] Allwood D A, Xiong G, Cooke M D, Faulkner C C, Atkinson D, Vernier N and Cowburn R P 2002 Science 296 2003-2006

[43] Zhu Y, Zhang Z, Ma B and Jin Q Y 2012 J. Appl. Phys. 11107 C106

[44] Wu S B, Yang X F, Chen S and Zhu T 2013 J. Appl. Phys. 113 17C119

[45] Kirchner H 1973 Rev. Sci. Instrum. 44 379-382

[46] Goa P E, Hauglin H, Olsen A A F, Baziljevich M and Johansen T H 2003 Rev. Sci. Instrum. 74 141-146

[47] Lange M, Guénon S, Lever F, Kleiner R and Koelle D 2017 Rev. Sci. Instrum. 88 123705

[48] McCord J 2015 J. Phys. D 48333001

[49] Goa P E, Hauglin H, Baziljevich M, Il'yashenko E, Gammel P L and Johansen T H 2001 Supercond. Sci. Technol. 14729

[50] Jiang W, Upadhyaya P, Zhang W, Yu G, Jungfleisch M B, Fradin F Y, Pearson J E, Tserkovnyak Y, Wang K L, Heinonen O et al. 2015 Science 349 283-286

[51] Collins-Mcintyre L J, Duffy L B, Singh A, Steinke N J, Kinane C J, Charlton T R, Pushp A, Kellock A J, Parkin S S, Holmes S N, Barnes C H, Van Der Laan G, Langridge S and Hesjedal T 2016 EPL 11527006

[52] Das D, Malik K, Deb A K, Dhara S, Bandyopadhyay S and Banerjee A $2015 \mathrm{~J}$. Appl. Phys. 118045102

[53] Roy A, Guchhait S, Dey R, Pramanik T, Hsieh C C, Rai A and Banerjee S K 2015 ACS Nano 9 3772-3779

[54] He H T, Wang G, Zhang T, Sou I K, Wong G K, Wang J N, Lu H Z, Shen S Q and Zhang F C 2011 Phys. Rev. Lett. 106166805

[55] Liu M, Zhang J, Chang C Z, Zhang Z, Feng X, Li K, He K, Wang L L, Chen X, Dai X, Fang Z, Xue Q K, Ma X and Wang Y 2012 Phys. Rev. Lett. 108036805

[56] Kim Y S, Brahlek M, Bansal N, Edrey E, Kapilevich G A, Iida K, Tanimura M, Horibe Y, Cheong S W and Oh S 2011 Phys. Rev. B 84073109

[57] Liu M, Chang C Z, Zhang Z, Zhang Y, Ruan W, He K, Wang L 1, Chen X, Jia J F, Zhang S C, Xue Q K, Ma X and Wang Y 2011 Phys. Rev. B 83165440

[58] Kim D, Syers P, Fuhrer M S, Paglione J and Butch N P 2013 Nat. Commun. 4 2040

[59] Kamboj V S, Singh A, Ferrus T, Beere H E, Duffy L B, Hesjedal T, Barnes C H and Ritchie D A 2017 ACS Photonics 4 2711-2718

[60] Soldatov I, Panarina N, Hess C, Schultz L and Schäfer R 2014 Phys. Rev. B 90 104423 